\title{
SOME REMARKS ON ALMOST PERIODIC TRANSFORMATIONS
}

\author{
P. ERDÖS AND A. H. STONE
}

In a recent paper in this Bulletin (see [3] ${ }^{1}$ ), W. H. Gottschalk has proved a number of interesting theorems on "recurrent" and "almost periodic" homeomorphisms of a space on itself. In the first part of the present note we give very simple proofs of some of Gottschalk's theorems in an even more general form. In the second half we consider "regular" transformations in more detail.

\section{Recurrent and almost periodic transformations.}

Notations. Let $f$ be a continuous mapping (not necessarily a homeomorphism) of a topological space $X$ in itself (that is, $f(X) \subset X)$. We say that $f$ is recurrent at a point $x \in X$, or that $x$ is recurrent un$\operatorname{der} f$, if, given any neighbourhood $U(x)$ of $x$, there exist infinitely many positive integers $n$ for which $f^{n}(x) \in U(x)$. (This definition is equivalent to Gottschalk's if $X$ is a $T_{1}$ space.) Further, $f$ is almost periodic at $x$ if, given any $U(x)$, there exists an $N(x, U(x))>0$ such that for the (infinite) sequence $\left\{n_{i}\right\}$ of positive integers for which $f^{n_{i}}(x) \in U(x)$ we have $n_{i+1}-n_{i} \leqq N$.

THEOREM I. If a continuous mapping $f$ of a topological space $X$ in itself is either (a) recurrent, or (b) almost periodic, at $x$, then so is $f^{k}$, for each positive integer $k .^{2}$

PRoof. Let $N_{r}$ denote the class of positive integers congruent to $r \bmod k$. We may clearly assume that one at least of the classes $N_{1}, N_{2}, \cdots, N_{k-1}$, say $N_{r}$, satisfies : every neighbourhood $U(x)$ of $x$ contains $f^{n}(x)$ for infinitely many values of $n \in N_{r}$; for otherwise each $U(x)$ will contain $f^{n}(x)$ for all large enough $n \in N_{k}$, and the theorem will follow trivially.

Now let $U_{0}$ be any given open set containing $x$. Choose $n_{1} \in N_{r}$ such that $f^{n_{1}}(x) \in U_{0}$. Since $f^{n_{1}}$ is continuous, there exists an open set $U_{1} \ni x$ such that $U_{1} \subset U_{0}$ and $f^{n_{1}}\left(U_{1}\right) \subset U_{0}$. Choose $n_{2} \in N_{r}$ such that $f^{2}(x) \in U_{1}$; and so on. In this way, we define integers $n_{1}, \cdots, n_{k-1}$ $\in N_{r}$ and open sets $U_{1} \supset U_{2} \supset \cdots \supset U_{k-1} \ni x$ such that $f^{n_{i}}(x) \in U_{i-1}$ and $f^{n_{i}}\left(U_{i}\right) \subset U_{i-1}$.

Received by the editors June 6, 1944 .

1 Numbers in brackets refer to the bibliography at the end of the paper.

2 Theorem I(a) is Theorem 1 of [3], without the restriction that $f$ be a homeomorphism. Theorem $\mathrm{I}(\mathrm{b})$ is Theorem 6 of [3], without the restrictions that $f$ be a homeomorphism and that $X$ be compact. 
In case (a), we observe that for infinitely many integers $m \in N_{r}$ we have $f^{m}(x) \in U_{k-1}$. For each such $m$, consider $M=n_{1}+n_{2}+\ldots$ $+n_{k-1}+m$; clearly $M \equiv 0 \bmod k$, and it is easy to see that $f^{M}(x) \in U_{0}$. Since there are infinitely many values of $M$, and $U_{0}$ was any open set containing $x$, this proves that $f^{k}$ is recurrent at $x$.

In case (b), there will be an infinite sequence $\left\{m_{i}\right\}$ of positive integers (not necessarily in $N_{r}$ ) such that $f^{m_{i}}(x) \in U_{k-1}$ and $\sup \left(m_{i+1}-m_{i}\right)<\infty$. Now assume temporarily that $k$ is prime. Then, for each $m_{i}$, one of the $k$ incongruent integers $m_{i}, m_{i}+n_{k-1}, \cdots, m_{i}$ $+n_{k-1}+n_{k-2}+\cdots+n_{2}+n_{1}$ will be congruent to $0 \bmod k$; call this integer $M_{i}$. It is easy to see that $f^{M_{i}}(x) \in U_{0}$ and that $\sup \left(M_{i+1}-M_{i}\right)$ $<\infty$. On rearranging the sequence $\left\{M_{i}\right\}$ in increasing order of magnitude, we see that the theorem is proved in this case-if $k$ is prime. And the theorem follows in general, by induction over $k$.

THEOREM II. Let $f$ be a homeomorphism of a connected topological space $X$ on itself $(f(X)=X)$. If a point $x$, recurrent under $f$, separates two other recurrent points in $X$, then $f$ is periodic at $x .^{3}$

Proof. Let $X-(x)=A \cup B$, where $A, B$ are mutually separated sets and $A, B$ contain the recurrent points $y, z$ respectively. Thus $\bar{A}=A \cup(x), \bar{B}=B \cup(x)$, and the sets $\bar{A}$ and $\bar{B}$ are connected. If the theorem is false, then for every positive integer $n$ we have $f^{n}(x) \neq x$; from this we shall derive a contradiction.

We first show that a positive integer $k$ exists such that:

(1) $f^{k}(\bar{A})$ meets $\bar{A}$ and $f^{k}(\bar{B})$ meets $\bar{B}$.

For suppose not. Without loss of generality, we may assume that $f(x) \in A$. We assert that:

(2) $f^{n}(\bar{A})$ meets $A$, for every positive integer $n$.

For this is true when $n=1$, since $f(\bar{A}) \ni f(x)$. Suppose (2) is true when $n=m$. Then, since (1) is false, $f^{m}(\bar{B}) \cap \bar{B}=0$, and so $f^{m}(\bar{B}) \subset A$. Hence, since $f^{m}$ is a 1-1 mapping, $f^{m}(A) \supset \bar{B}$, so that $f^{m+1}(\bar{A}) \supset f^{m+1}(A)$ $\supset f(\bar{B}) \ni f(x) \in A$. Thus (2) follows for $n=m+1$, and therefore holds for all $n$, by induction. Hence, since (1) is false, we have from (2) that $f^{n}(\bar{B}) \cap \bar{B}=0$ for every positive integer $n$. This contradicts the fact that $f$ is recurrent at $z \in B$; and so (1) is proved.

Again without loss of generality, we may assume now that $f^{k}(x) \in A$. Thus $f^{k}(\bar{B})$ meets $A$; but, from (1), $f^{k}(\bar{B})$ also meets $\bar{B}$, and therefore (being connected) contains $x$. Since $f^{k}$ is $1-1$, we thus have $x \notin f^{k}(A)$, and therefore $x \notin f^{k}(\bar{A})$. Therefore the connected set

${ }^{3}$ Essentially [3, Theorem 2], without compactness restrictions. The proof as given assumes $(x)$ is closed, but could easily be modified so as to dispense with this assumption. 
$f^{k}(\bar{A})$, which meets $A$ (in $f^{k}(x)$ at least), cannot meet $\bar{B}$, so that $f^{k}(\bar{A}) \subset A$. It readily follows that, for every positive integer $n$, $f^{n k}(\bar{A}) \subset f^{k}(\bar{A}) \subset A$. Since $f^{k}(\bar{A})$ is a closed set not containing $x$, this contradicts the fact that (by Theorem I) the mapping $f^{k}$ is recurrent at $x$.

2. Regular and strongly almost periodic transformations. Now let $X$ be a metric space. A mapping $f(X) \subset X$ is said to be strongly almost periodic (cf. [3]-or "almost periodic," in [1]) if, given $\epsilon>0$, there exists an integer $L>0$ such that every set of $L$ consecutive positive integers contains an $n$ satisfying $\rho\left(x, f^{n}(x)\right)<\epsilon$ for all $x \in X$.

THEOREM III. If $X$ is a totally bounded metric space, and if $f$ is a homeomorphism of $X$ in itself all of whose negative powers are equiuniformly continuous, then $f$ is strongly almost periodic."

Proof. By hypothesis, given $\epsilon>0$, there exists a $\delta>0$ such that $\rho\left(f^{-m}(x), f^{-m}(y)\right)<\epsilon / 2$ whenever $m$ is a positive integer and $x, y$ are points of $f^{m}(X)$ such that $\rho(x, y)<\delta$. We may clearly assume that $\delta<\epsilon / 2$.

Let $A_{1}, \cdots, A_{r}$ be a finite covering of $X$ by sets of diameter less than $\delta$. For each positive integer $m$ we define a square matrix $B(m)$ of order $r$ by setting $b_{i j}(m)=1$ if $f^{m}\left(A_{i}\right)$ meets $A_{j}, 0$ otherwise. Of all the matrices $B(1), B(2), \cdots$, only a finite number can be distinct; let $B(1), \cdots, B(L)$ include all the distinct matrices $B(m)$. We shall prove the theorem by showing that, given any positive integer $M$, an integer $n$ exists such that: (a) $M \leqq n<M+L$; (b) $\rho\left(x, f^{n}(x)\right)<\epsilon$, for all $x \in X$.

In fact, $B(M+L)=B(m)$ for some $m \leqq L$. Define $n=M+L-m$; thus (a) is certainly true. To verify (b), let $x \in X$ be given, and suppose $x \in A_{i}, f^{M+L}(x) \in A_{j}$. Then $b_{i j}(M+L)=1$, and so $b_{i j}(m)=1$ also; thus $f^{m}\left(A_{i}\right)$ meets $A_{j}$. Let $y \in A_{j} \cap f^{m}\left(A_{i}\right)$. Then $\rho\left(x, f^{n}(x)\right)$ $\leqq \rho\left(x, f^{-m}(y)\right)+\rho\left(f^{-m}(y), f^{M+L-m}(x)\right) \leqq \delta\left(A_{i}\right)+\delta\left(f^{-m}\left(A_{j}\right)\right)$ (where $\delta(A)$ denotes the diameter of $A)<\delta+\epsilon / 2<\epsilon$. Q.E.D.

A homeomorphism $f$ of a metric space in itself is said to be regular if all its powers (positive or negative) are equi-uniformly continuous. Thus every isometry is regular; and conversely it is easy to see that if a regular homeomorphism $f$ maps the space on itself (as it must if the space is compact), then the space can be remetrized so that $f$ becomes an isometry.

THEOREM IV. Let $f$ be a regular homeomorphism of a totally bounded

4 This is closely related to Theorems 5 and 6 of [2, pp. 701, 702]. It would be desirable to weaken the equi-continuity assumption on the powers of $f$. 
metric space in itself. There exists an increasing sequence $\left\{n_{i}\right\}$ of positive integers such that $f^{n^{i}}(x) \rightarrow x$ and $f^{\left(n_{i}\right)^{2}}(x) \rightarrow x$, uniformly, for all $x \in X^{5}$

Proof. Given $\epsilon>0$, we must show that arbitrarily large integers $n$ exist such that, for all $x \in X, \rho\left(f^{n}(x), x\right)<\epsilon$ and $\rho\left(f^{n^{2}}(x), x\right)<\epsilon$. Now, from Theorem III, there exists a sequence $\left\{m^{\prime}\right\}$ such that $f^{m^{\prime}}(x) \rightarrow x$ uniformly. We shall first show that a sufficiently rapidly increasing subsequence $\left\{m_{i}\right\}$ of $\left\{m^{\prime}\right\}$ will satisfy (for all $x \in X$, and every pair of integers $r, s$ with $0<r<s$ ) both

$$
\rho\left(f^{\left(m_{r}+m_{r}+1+\cdots+m_{s}\right)^{2}}(x), \quad f^{m_{r}^{2}+m_{r}^{2}+1+\cdots+m_{s}^{2}}(x)\right)<\epsilon / 2
$$

and

$$
\rho\left(f^{\left(m_{r}+m_{r}+1+\cdots+m_{s}\right)}(x), x\right)<\epsilon .
$$

For, by hypothesis, there is a positive function $\delta(\epsilon)$ of the positive variable $\epsilon$ such that $\rho\left(f^{m}(x), f^{m}(y)\right)<\epsilon / 2$ whenever $\rho(x, y)<\delta(\epsilon)$ and $f^{m}(x), f^{m}(y)$ are defined $(m=0, \pm 1, \pm 2, \cdots)$. Choose $m_{1}$ to be an $m^{\prime}$ so large that $\rho\left(f^{m_{1}}(x), x\right)<\epsilon / 2$ for all $x \in X$. Then define $m_{s}$ inductively to be an $m^{\prime}$ so large that: (a) $\rho\left(f^{m_{s}}(x), x\right)<\epsilon / 2^{s}$, and (b) $\rho\left(f^{2\left(m_{r}+\cdots+m_{s-1}\right) m_{s}}(x), x\right)<\delta\left(\epsilon / 2^{s}\right)$, for all $x \in X$ and every $r$ between 1 and $s-1$. Clearly (2) follows from (a); and (1) follows from (b), since (by the triangle law)

$$
\begin{aligned}
& \rho\left(f^{\left(m_{r}+m_{r}+1+\cdots+m_{s}\right)^{2}}(x), f^{m_{r}^{2}+m_{r+1}^{2}+\cdots+m_{s}^{2}}(x)\right)
\end{aligned}
$$

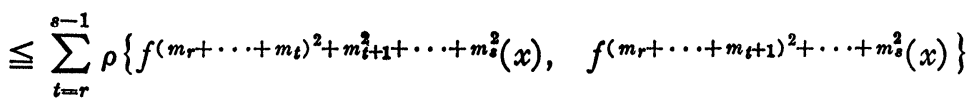

$$
\begin{aligned}
& <\sum \epsilon / 2^{t+2}<\epsilon / 2 \text {. }
\end{aligned}
$$

Now let $A_{1}, \cdots, A_{k}$ be a finite covering of $X$ by sets of diameter less than $\min \{\delta(\delta(\epsilon)), \delta(\epsilon) / 2\}$; and, as in the proof of Theorem III, let $B(n)$ denote the square matrix of order $k$ formed by setting $b_{i j}(n)$ $=1$ if $f^{n}\left(A_{i}\right)$ meets $A_{j}, 0$ otherwise. Consider the sequence of matrices

$$
B\left(m_{1}^{2}\right), B\left(m_{1}^{2}+m_{2}^{2}\right), \cdots, B\left(m_{1}^{2}+m_{2}^{2}+\cdots+m_{t}^{2}\right), \cdots .
$$

For some two integers $r, s$ with $0<r<s$ we must have $B\left(m_{1}^{2}+\cdots+m_{r}^{2}\right)$ $=B\left(m_{1}^{2}+\cdots+m_{s}^{2}\right)$. An easy calculation (cf. the end of the proof of Theorem III) now shows that, for all $x \in X, \rho\left(f^{m_{1}^{2}+\cdots+m_{r}^{2}}(x)\right.$,

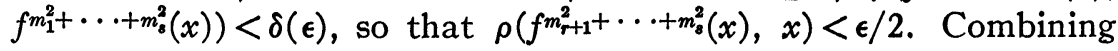

$\checkmark$ This is essentially a generalization of a theorem of Hardy and Littlewood on the denseness of the fractional parts of $\left\{n^{2} \alpha\right\}, \alpha$ irrational. See [4, p. 157]. It would be desirable to weaken the hypothesis of regularity. 
this with (1), and writing $n=m_{r+1}+\cdots+m_{s}$, we see thet $\rho\left(f^{n^{2}}(x), x\right)<\epsilon$. Since we also have $\rho\left(f^{n}(x), x\right)<\epsilon$, from (2), the theorem is proved.

In the same way (using induction over $k$ ) one could prove that, given any positive integer $k$, there exists an increasing sequence $\left\{n_{i}\right\}$ of positive integers for which, simultaneously, $f^{n_{i}}(x) \rightarrow x, f^{n_{i}^{2}}(x)$ $\rightarrow x, \cdots, f^{n_{i}^{k}}(x) \rightarrow x$, uniformly, for all $x \in X$. In fact, substantially the same argument will show that this holds if each power $n^{t}$ $(t=1, \cdots, k)$ is replaced by any polynomial $\phi_{t}(n)$ of degree $t$, having integer coefficients, positive leading coefficient, and zero constant term.

These results provide partial answers to the question, raised by Theorem I, as to what can be said about the sequence of integers $n$ for which $f^{n}(x)$ is in a given neighbourhood of $x, f$ being recurrent at $x$. Thus, under the hypotheses of Theorem IV, this sequence contains infinitely many squares, and in fact infinitely many $k$ th powers. Query: Will it (under "reasonable" hypotheses-for example, nonperiodicity) contain infinitely many primes? (For the special case in which $X$ is the real line mod 1 , and $f(x)=x+\alpha$, where $\alpha$ is a fixed irrational number, the answer is affirmative, though the proof is difficult. $\left.{ }^{6}\right)$

\section{BIBLIOGRAPHY}

1. R. H. Cameron, Almost periodic transformations, Trans. Amer. Math. Soc. vol. 36 (1934) pp. 276-291.

2. Ky Fan, Les fonctions asymptotiquement presque-périodiques . . , Math. Zeit. vol. 48 (1943) pp. 685-711.

3. W. H. Gottschalk, Powers of homeomorphisms with almost periodic properties, Bull. Amer. Math. Soc. vol. 50 (1944) pp. 222-227.

4. G. H. Hardy and J. E. Littlewood, Some problems of Diophantine approximation, Acta Math. vol. 37 (1914) pp. 155-190.

5. P. Turan, Über die Primzahlen der arithmetischen Progressionen, Acta Univ. Szeged. vol. 8 (1936-1937) pp. 226-235.

6. I. M. Vinogradoff, New estimations of trigonometric sums containing primes, C. R. (Doklady) Acad. Sci. URSS. vol. 17 (1937) pp. 165, 166.

\section{Purdue University}

\footnotetext{
${ }^{6}$ This follows from results of Vinogradoff; cf. [6]. See also [5, p. 234].
} 